\title{
ARTÍCULOS PRIMARIOS
}

\section{CARACTERIZACIÓN DE LA ESTRUCTURA HISTOLÓGICA DE LOS ÓRGANOS LINFOIDES EN ALPACAS (Lama pacos)}

\author{
Daniel Fernández T. ${ }^{1}$, Luis Tabacchi N. ${ }^{2}$, Nieves Sandoval Ch.', \\ Rosa Perales C. ${ }^{2}$ y Gilberto Santillán $\mathrm{A}^{2}$
}

\section{Nhstrat:}

The anatomic structure of lymphatic system organs ( 12 different lymph nodes, thymus, Peyer's patches and spleen) from 10 alpacas ( 5 female and 5 male) aged 2-8 years, is described. Histological sections were prepared utilizing hematoxylin-eosin (for complete cellular distribution), Verhoeff (for collagen) and Gomori (for reticular fibers) stains. The lymphatic organs of the alpaca were found to be similar to those of other mammals although a few exceptions exist. The lymph nodes are pseudolobulate and have an irregular surface. Histologically they do not exhibit a marked difference between the cortical and medullar regions. The thymus is visible in adult alpacas and consists of three successive lobes, with the larger ones subdivided into smaller lobes which are in turn composed of lobules. Peyer's patches were macroscopically visible in 4 of the 8 alpacas examined, but microscopic analysis revealed the presence of isolated lymphatic nodules in all the animals, indicating that they are permanent structures. The spleen capsule is thick and isolated smooth muscle fibers observed in the red pulp.

Key Words: Alpaca, lymphatic system organs, lymph node, thymus, Peyer's patches, spleen

\section{Resumen}

El objetivo del presente trabajo fue el estudió anatómo-histológico de los órganos linfoides de 10 alpacas, 5 hembras y 5 machos entre los 2 y 8 años de edad: Ganglios linfáticos, bazo, timo, placas de Peyer. El estudio histológico se realizó usando 3 técnicas de coloración: Hematoxilina - Eosina (Distribución celular), Verhoeff (Para fibras colágenas) y Gomori (Para fibras reticulares). Anatómicamente los ganglios linfáticos son irregulares superficialmente y seudolobulados. El timo fue encontrado aún en alpacas adultas y muestran tres lobulaciones sucesivas, los lóbulos mayores están conformados por sublóbulos medianos interdependientes que a su vez están conformados por los lobulillos tímicos. Las placas de peyer muestran diferentes dimensiones entre alpacas siendo imperceptibles en algunos animales. Histológicamente los ganglios linfáticos son similares a los de los demás mamíferos, sin embargo no muestran gran diferenciación entre corteza y médula, además los senos subcapsulares ocupan ciertos sectores bajo la cápsula y se continúan con áreas parenquimatosas de menor densidad celular que se 
disponen desde la cápsula hasta el hilio con presencia de senos linfáticos en su trayecto. Las vénulas endoteliales altas (vénulas especializadas) se distribuyen por todo el parénquima. El bazo muestra la pulpa roja y pulpa blanca característicos, la cápsula es gruesa compuesta de tres capas siendo la capa conjuntiva más delgada que la capa muscular que a su vez está conformada por dos estratos, además se observan fibras musculares lisas aisladas en la pulpa roja. En los sublóbulos del timo, los lobulillos tímicos típicos son confluyentes a través de la porción medular. Las placas de Peyer son estructuras permanentes histológicamente

Palabras clave: Alpaca, ganglio linfático, bazo, timo, placa de peyer.

\section{Introduectón}

Las especies superiores cuentan con un complicado sistema de vigilancia y defensa capaz de responder a agentes extemos o internos que posean capacidad inmunogénica. Este sistema está conformado principalmente por linfocitos agrupados en órganos especializados que, dependiendo de su función se clasifican en: órganos fuente de células linfoides, órganos linfoides primarios y órganos linfoides secundarios (Roitt, 1997; Rojas, 1995; Tizard, 1992). En los órganos linfoides primarios se realiza mayormente la linfopoyesis y maduración linfocitaria, está conformado por la médula ósea, el timo, la bursa de Fabricio (en aves) y algunas placas de Peyer. La función de los órganos linfoides secundarios es la de proporcionar un entomo óptimo de interacción entre el antígeno las células linfoides y las células accesorias, y estos órganos son los ganglios linfáticos, el bazo, las placas de Peyer, los ganglios hemolinfáticos y el tejido linfoide asociado a la lámina propia de los sistemas respiratorio, urogenital y digestivo (Banks, 1992; Ross, 1994; Tizard, 1992). Los complicados factores y mecanismos del sistema inmune se desarrollan dentro de los órganos linfoides y se reflejan en la modificación de la morfología estructural de dichos órganos (Kimura, 1996 y Girard, 1995).

La conformación histológica de los órganos linfoides ha sido ampliamente estudiada en las especies domésticas, pero muy poco en alpacas, por lo tanto es necesario realizar una caracterización detallada de las estructuras componentes de cada órgano linfoide, las mismas que poseen gran importancia intrínseca y permiten comprender mejor los mecanismos fisio-patológicos de la inmunidad. En tal sentido, el presente trabajo complementa y amplia la información hasta ahora obtenida en el ámbito de la investigación científica respecto al sistema inmunológico de la alpaca.

\section{Materiales y Métodos}

Se emplearon 10 alpacas, 5 machos y 5 hembras, aparentemente sanas provenientes de la localidad de Pinaya a $5000 \mathrm{msnm}$, en el departamento del Cuzco. La edad aproximada fue determinada observando las piezas dentarias y corresponden a 2 años $(n=2), 3$ años ( $n=1), 5$ años ( $n=3), 7$ años $(n=2)$ y 8 años $(n=2)$.

Los animales fueron sacrificados, e inmediatamente se procedió a recolectar los órganos linfoides. En base a su importancia como principales centros linfáticos, colectaron:

- Ganglios Linfáticos: Retrofaríngeo lateral, submandibular, cervical superficial, inguinal superficial, poplíteo, mediastínico anterior, mediastínico medio.

- Traqueobronquial posterior, duodenal, yeyunal, ileal, cecocólico. Estos ganglios fueron tomados íntegros.

- Bazo: Secciones de tejido de $0.5 \mathrm{~cm}$ de grosor. 
- Timo: Secciones de tejido de $0.5 \mathrm{~cm}$ de grosor.

- Placas de Peyer: Secciones de duodeno, yeyuno e ileon, de $3 \mathrm{~cm}$ de longitud conteniendo dichas estructuras linfoides. De los animales en los que no se pudo observar placas de Peyer macroscópicamente, se tomaron al azar secciones de $3 \mathrm{~cm}$ de longitud de cada porción intestinal.

Se emplearon tres técnicas de coloración: La coloración de Hematoxilina Eosina muestra la distribución del estroma y parénquima en los órganos mencionados, la coloración de Verhoeff muestra la distribución de las fibras colágenas y elásticas y la coloración de Gomori muestra la distribución de las fibras reticulares.

\section{Resultades}

\section{Descripción Anatómica}

Para ubicar los órganos linfoides en las alpacas, se tomó como referencia la descripción anátomotopográfica realizada por Carrasco (1968) y Gambirazio (1967) acerca de la ubicación de los principales centros linfáticos del miembro pélvico y del miembro anterior de la alpaca. Los hallazgos anatómicos más importantes en los órganos linfoides son:

\section{Ganglios linfáticos}

Los ganglios linfáticos de las Alpacas son entidades únicas, reconocibles y su ubicación es similar a la de los demás rumiantes, exceptuando algunas peculiaridades.

Principalmente los ganglios externos, muestran una superficie irregular por la presencia de hendiduras que dan al órgano la apariencia de ser lobulado. Estas hendiduras dificultan la ubicación del hilio (Fig. 1). La disposición del hilio dentro del ganglio es variable, siendo bastante profunda en los ganglios externos y muy pequeña en los ganglios internos.

El retrofaringeo lateral fue ubicado ventrolateralmente al ala del atlas y borde caudal de la glándula parótida; el submandibular está localizado en el ángulo

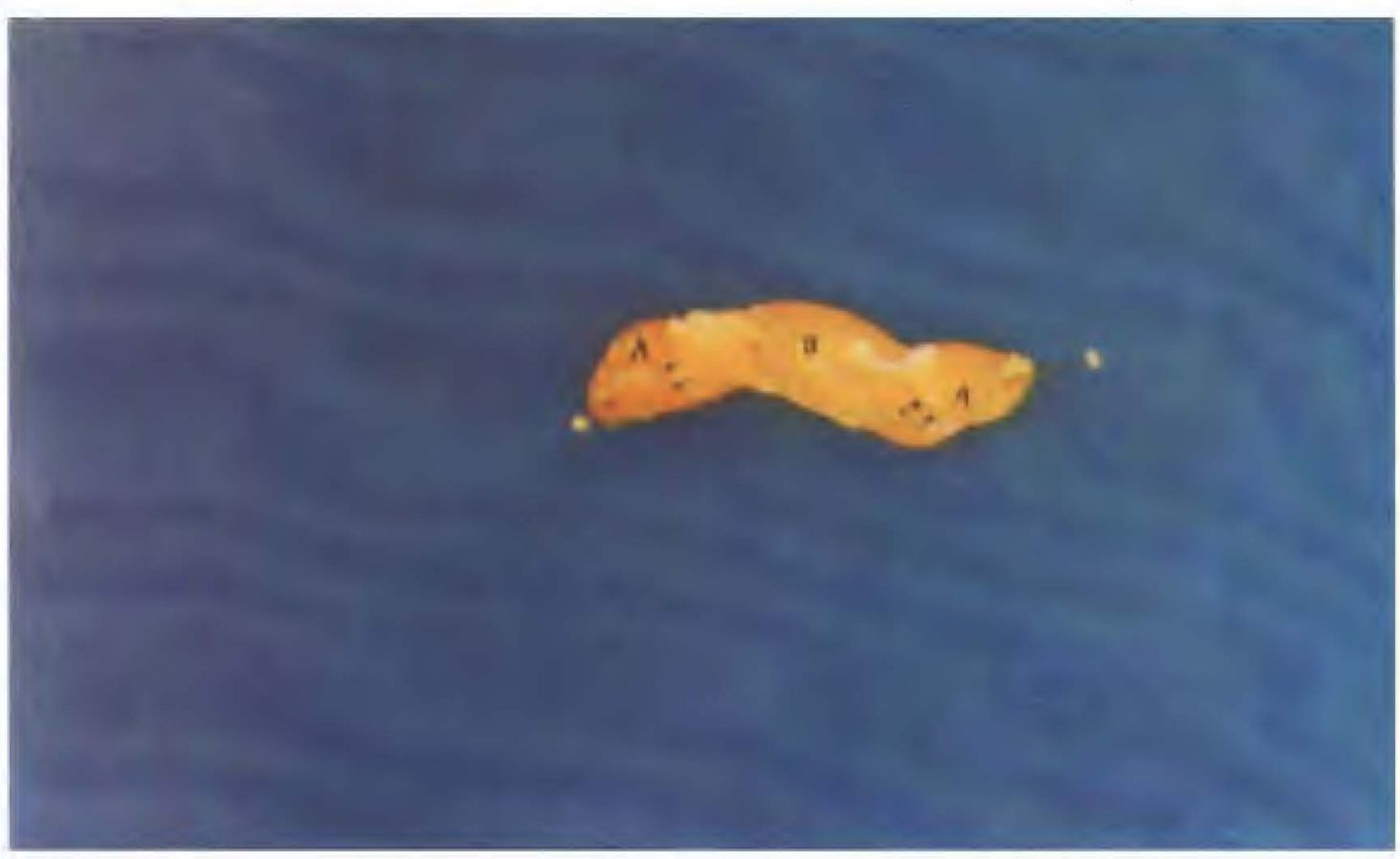

Figura 1. Ganglio Inguinal. A. Seudolobulaciones. B. Hilio. 
de la mandíbula, anterior a la glándula parótida y cubierto por el músculo depresor del labio inferior; el cervical superficial en la unión del cuello con el miembro anterior, por delante del músculo pectoral profundo porción anterior y relacionado al ángulo craneal de la escápula, cubierto por el músculo omotransverso, braqueocefálico y trapecio cervical.

El ganglio inguinal superficial en la región inguinal cerca al anillo inguinal superficial y lateralmente a la glándula mamaria (hembras) o al prepucio (machos), cubierto por tejido adiposo, es alargado y puede constar de una o dos unidades separadas por lado; el poplíteo está relacionado al borde caudal del músculo gastronemio y cubierto por los músculos biceps femoral y semitendinoso, caudal a los nervios peroneo común y tibial.

Los ganglios linfáticos mediastínicos comprende a los mediastínicos anteriores, medios o traqueobronquiales y posteriores. Los mediastínicos anteriores, ubicados cerca de la aurícula izquierda, asociados a los grandes vásos, anteriores al cayado aórtico y próximos a la traquea y el esófago, son pequeños y ovalados. Los traqueobronquiales están adheridos a la traquea y muy cerca de su bifurcación, son un poco más voluminosos que los mediastínicos anteriores y constan de 2 a 3 unidades, alargados, aplanados lateralmente o algo arriñonados. Los mediastínicos posteriores constan de dos unidades, caudales al corazón y adheridos entre la arteria aorta torácica y cara dorso lateral del esófago antes de su ingreso por el hiato esofágico del diafragma, son alargados $y$ muestran pocas irregularidades en la superficie.

Los ganglios mediastínicos son de consistencia blanda respecto a los ganglios externos revisados y tienen la cápsula delgada. Puede ser dificultoso ubicar a los ganglios mediastínicos anteriores y traqueobronquiales. Es difícil ubicar el hilio externamente y su extensión dentro del parénquima es bastante pequeña y estrecha.

Los ganglios mesentéricos se ubican en el mesenterio intestinal, cubiertos por tejido adiposo e incluidos dentro del peritoneo visceral, toman diferentes denominaciones según su relación con la porción intestinal correspondiente. Estos ganglios exceptuando a los cecocólicos, al igual que los mediastínicos, son de consistencia blanda respecto a los ganglios linfáticos externos y el hilio es pequeño y estrecho por lo que es difícil ubicarlo extemamente.

Los ganglio mesentéricos duodenales son generalmente pequeños ovalados y algo alargados, los mesentéricos yeyunales constan de varias unidades uno de los cuales es prominente y bastante alargado, aunque en algunos casos se presenta dividido en dos porciones separadas comunicadas por vasos linfáticos. Son algo aplanados lateralmente y la superficie irregular muestra hendiduras que forman seudo lobulaciones. Los mesentéricos ileales son de tamaño mediano, pocos en número respecto a los ganglios yeyunales, ovalados alargados y algunos son deformes. Los cecocólicos constan de tres a cuatro unidades muy cercanas entre sí, siendo una de ellas más grande que las otras. Son ovaladas y alargadas.

\section{Timo}

Es más voluminoso en animales jóvenes que en los animales adultos. Ubicado a la entrada del tórax en el mediastino anterior, conformado por lóbulos rodeados de una cápsula delgada. Los lóbulos mayores están conformados por sub-lóbulos medios, a su vez estos sub-lóbulos están divididos en los lobulillos tímicos los cuales pueden observarse con cierta dificultad.

\section{Placas de Peyer}

De los 10 animales solo 4 de ellos mostraron placas de Peyer macroscópicamente (a simple vista), y estas se distribuyen en todo el trayecto intestinal, pero fueron más 
evidentes en la porción ileocecal. En el duodeno y yeyuno son principalmente pequeñas (de $2 \mathrm{~mm}$ a $2 \mathrm{~cm}$ de diámetro). En el ileon son más numerosas, grandes y ovaladas (de diámetros de $1.5 \mathrm{a} 2 \mathrm{~cm}$ ). A nivel del ciego se presentan mucho más prominentes e irregulares (hasta $5 \mathrm{~cm}$ de largo). En el colon espiral son menos numerosos y más pequeños hasta casi desaparecer a nivel del recto.

\section{Ganglios Hemolinfáticos}

En la alpaca no se ha logrado ubicar alguna estructura compatible con los ganglios hemolinfáticos en las zonas anátomo topográficas descritas para los demás rumiantes.

\section{Descripción Histológica}

Se realizó el estudio histológico de 12 ganglios linfáticos distintos en cada alpaca, bazo, nódulos linfáticos asociados al intestino, y el timo.

\section{Ganglios Linfáticos}

Las características histológico estructurales comunes son:

- Los ganglios linfáticos están conformados por un estroma y parénquima típicos. Las células linfoides están empotradas en una red conformada por fibras y células reticulares (Fig, 2)

- Los senos subcapsulares se ubican en algunos sectores bajo la cápsula, así como los senos trabeculares rodean a algunas trabéculas. En gran medida el estroma se halla adherido al parénquima sin dejar espacios visibles.

- El parénquima muestra los folículos linfoides primarios y secundarios, estos últimos mas voluminosos y abundantes, conteniendo un centro germinal. También se observan áreas irregulares en donde las células se agrupan densamente, así como áreas en donde las células están mas disgregadas observándose el tejido reticular de sostén. Estos sectores de menor densidad celular tienen una disposición irregular y se extienden desde la cápsula hasta el hilio, conteniendo a los senos linfáticos. En el parénquima no es notoria la diferenciación entre corteza y médula, sin embargo, generalmente se observa las áreas celulares de mayor densidad en mayor concentración hacia la periferia y las áreas celulares menos densas y con pocos folículos hacia el hilio, sin llegar a configurar la imagen corticomedular esperada.

- Los senos linfáticos son espacios de amplitud variable y se ubican en todo el parénquima, principalmente en las áreas de menor densidad celular. Están tapizados por un endotelio plano simple y periféricamente son rodeados por una ligera condensación de fibras reticulares.

- Los vasos sanguíneos del hilio, se ramifican en arterias trabeculares de mediano calibre, las que a su vez abandonan la trabécula y se ramifican profundamente en el parénquima en arteriolas cuya ramificación formará las vénulas especializadas denominadas: vénulas de endotelio alto las cuales se distribuyen en todo el parénquima (Fig. 3),

\section{Hallazgo particular al Ganglio Cervical.}

Hacia un sector del parénquima se observan unas estructuras peculiares a manera de red tridimensional de cordones o tabiques, gruesos y confluyentes, que infiltran el parénquima y se muestran preferentemente rodeando a las trabéculas o a grupos de linfocitos. Están compuestas de tejido conjuntivo con una sustancia acidófila amorfa homogénea que contiene a pocas células fusiformes, pocas fibras colágenas y no se observan fibras reticulares ni elásticas, tiende a formar pequeños vacíos. Estas estructuras son más evidentes en animales de mayor edad. 


\section{Fernández et al.}

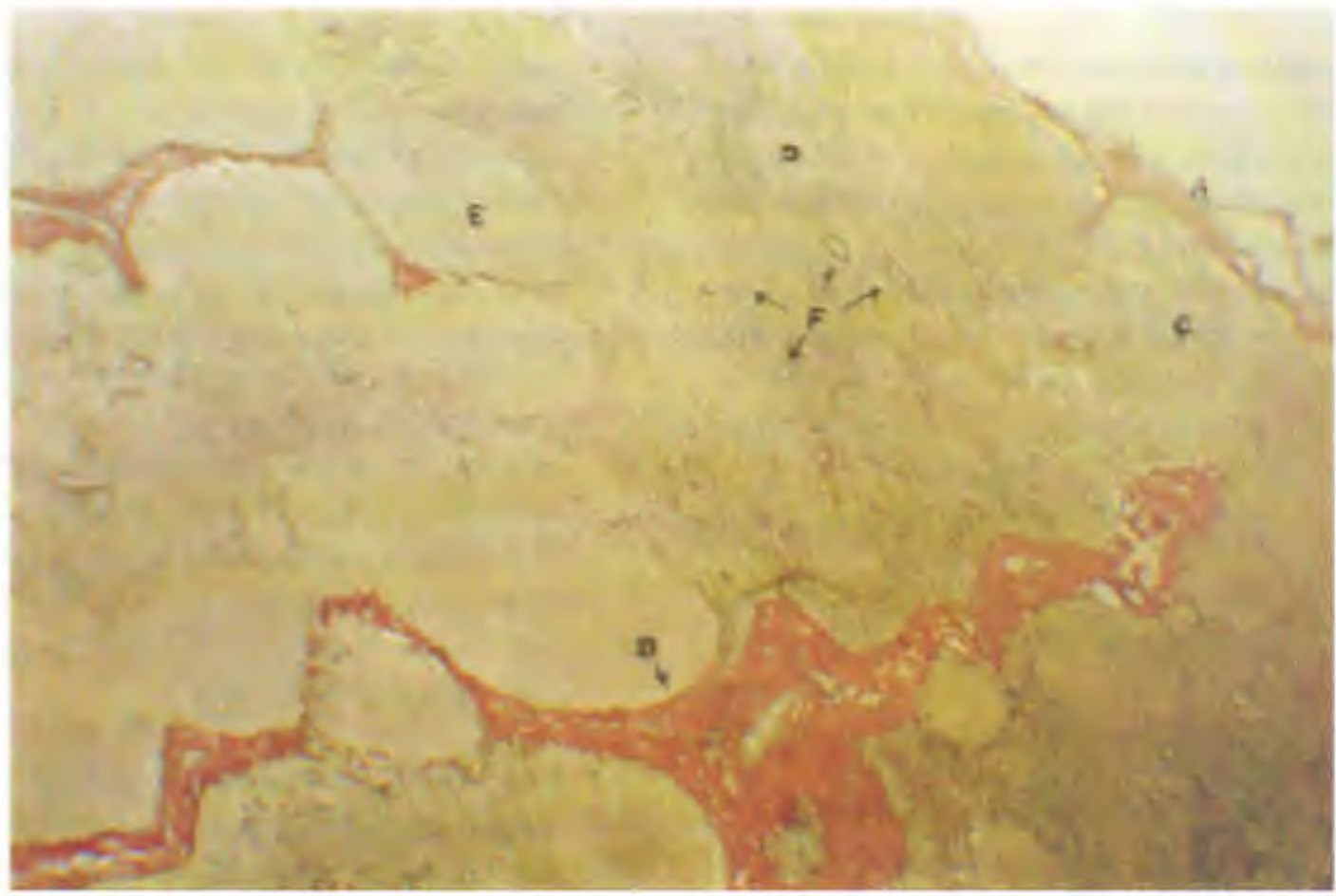

Figura 2. Estructura estromal de un ganglio linfático. Coloración de Gomoris. 5X. A: cápsula; B: trabéculas; C: fibras reticulares de las áreas de menor densidad celular; D: fibras reticulares del folículo primario; E: fibras reticulares del folículo sencundario; F: fibras reticulares de las vénulas de endotelio alto.

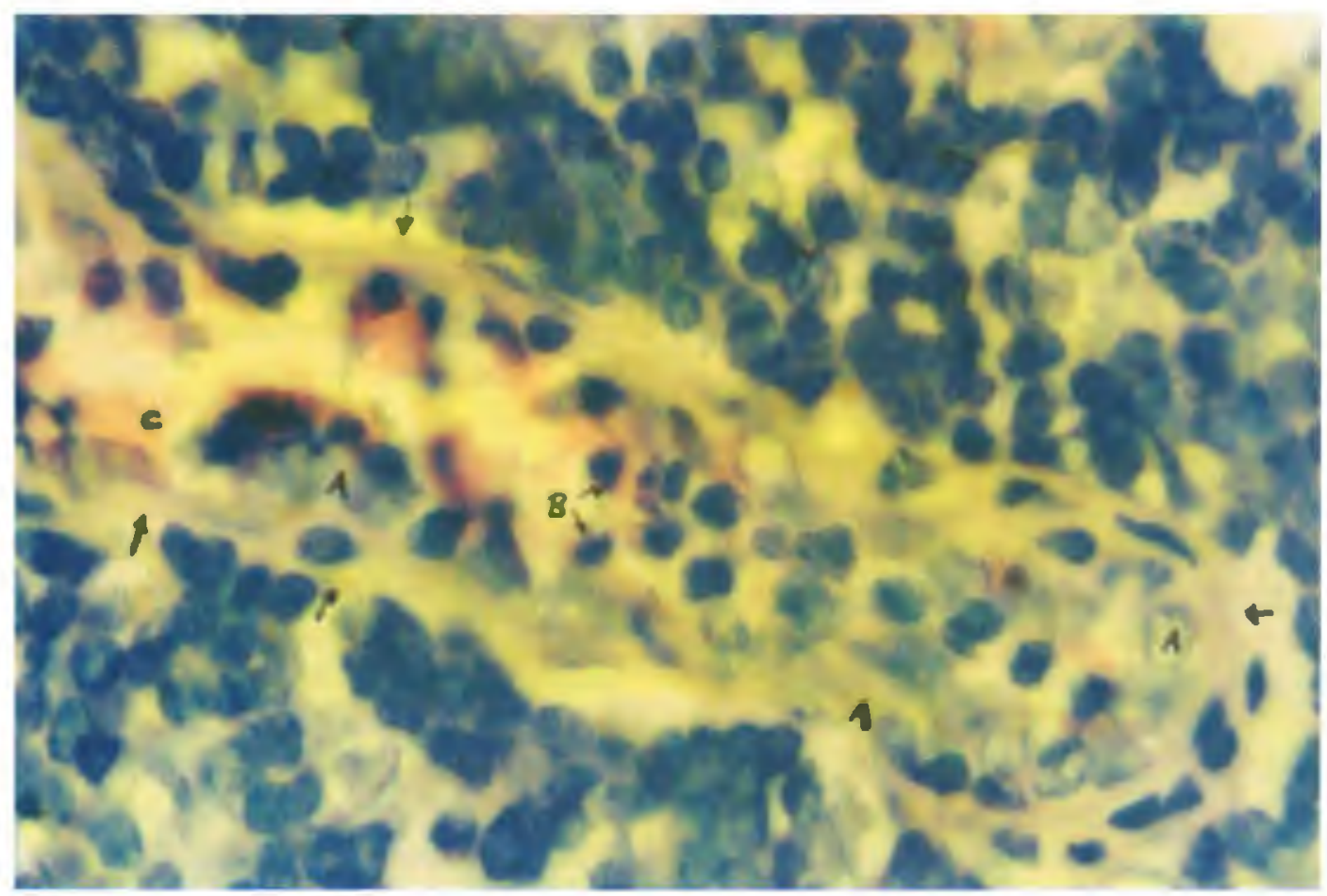

Figura 3. Vénula de endotelio alto de ganglio linfático. Coloración H-E. 100X. (flechas) A: endotelio alto, B: linfocitos; C: eritrocitos. 
Bazo

En el bazo la cápsula es bastante gruesa y compuesta por tres estratos: Estrato mesotelial, estrato conjuntivo, el cual constituye aproximadamente la cuarta parte del grosor total, y el estrato muscular que es el más grueso y está conformada por dos subcapas de musculo liso. Las trabéculas muestran grosor y longitud variable conformadas por fibras musculares lisas, fibras colágenas y pocas fibras reticulares entrelazadas dispuestas en sentido longitudinal. Presencia de pequeños haces de fibras musculares lisas aisladas en el parénquima.

El parénquima esplénico presenta la pulpa roja y la pulpa blanca característicos. La pulpa blanca está conformada por los folículos linfoides con centro germinal y por las vainas linfáticas periarteriales. La pulpa roja muestra gran concentración de eritrocitos lo cual hace difícil diferenciar a los cordones de Billroth (Fig. 4). Todo el parénquima presenta una red de fibras reticulares entre cuyas fibras se ubican las células linfoides y eritrocitos.
Timo

La cápsula es delgada, envuelve a los lobulos mayores e incluye a los sub-lóbulos medios. Cada sub-lóbulo a su vez posee una cápsula muy delgada adherida al parenquima. Las trabéculas se extienden desde la cápsula de cada sublóbulo y delimitan las porciones corticales del lobulillo tímico mas no la médula.

En el parénquima las unidades básicas son los lobulillos tímicos, los cuales conforman los sub-lóbulos medianos, los que a su vez formarán los lóbulos mayores. Cada lobulillo consta de una corteza periférica densa y una médula de posición central de células mas laxamente dispuestas entre las cuales se puede observar a las células reticuloepiteliales. En un sublóbulo medio los lobulillos tímicos son confluyentes a través de la porción medular. A nivel de la médula se pueden observar los corpúsculos de Hasall. Los vasos sanguíneos de pequeño calibre están rodeados por células retículo epiteliales conformando la barrera hematotímica.

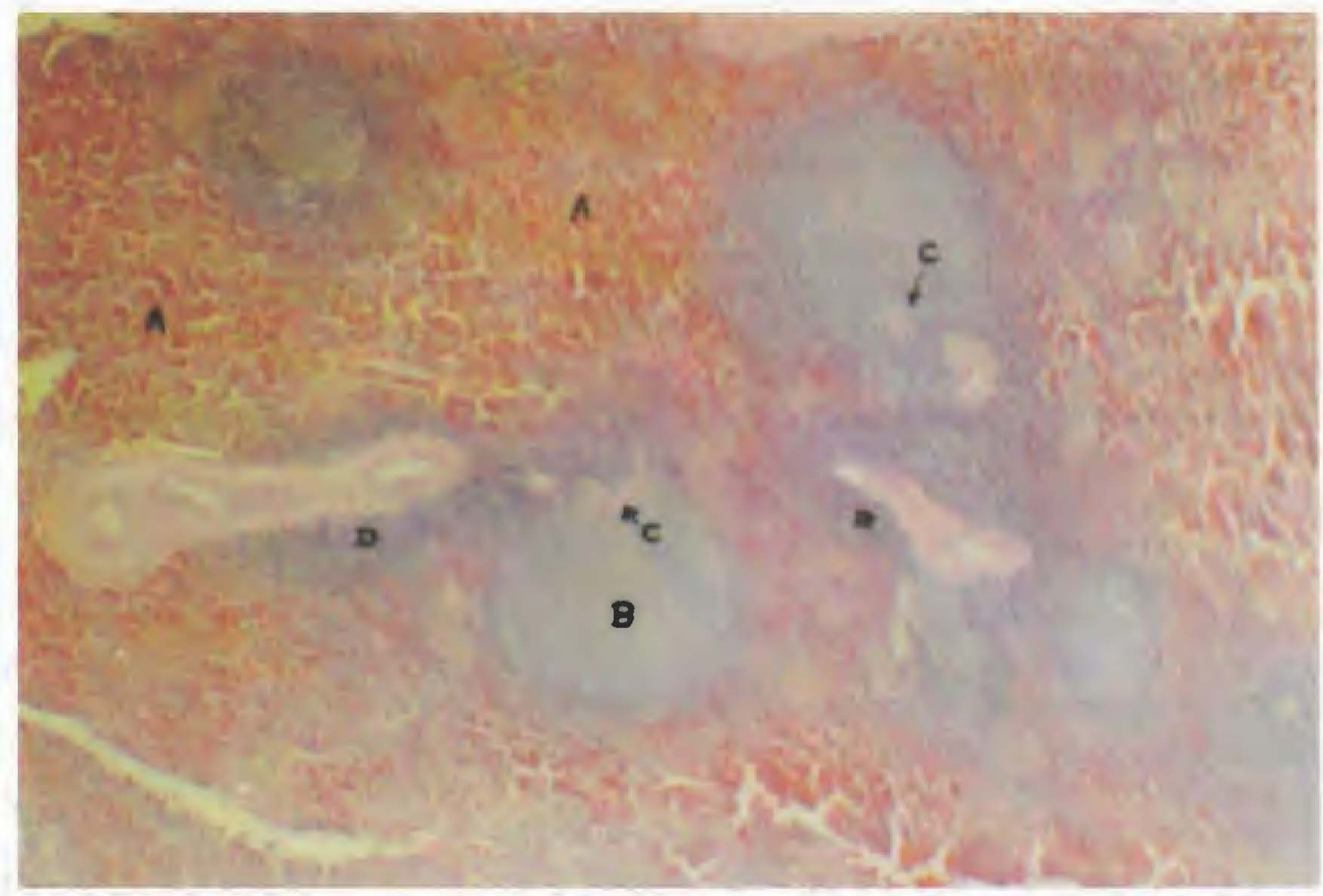

Figura 4. Bazo. Coloración H-E. 5X. A: pulpa roja; B: pulpa blanca: nódulo típico con centro germinal; C: arteriola central; D: Vaina linfática periarterial. 


\section{Tejido Linfoide Asociado al Intes- tino}

Los folículos linfáticos aislados y las placas de peyer, están ubicados en la submucosa de todo el trayecto intestinal, bajo la muscular de la mucosa la misma que muestra interrupciones en su continuidad permitiendo un contacto más íntimo de la estructura linfoide con la lámina propia de la mucosa a la cual infiltran. Dicha infiltración se presenta a veces desplazando a las vellosidades o solo infiltrando el corion de las mismas. Los folículos linfoides aislados y aquellos que conforman las placas, generalmente muestran un centro germinal. La irrigación se extiende desde la submucosa, los vasos penetran periféricamente la estructura nodular para formar las vénulas especializadas denominadas vénulas de endotelio alto, similares a las vénulas del ganglio linfático.

\section{Discusión}

Los órganos linfoides de las alpacas son similares a los de las demás especies sin embargo presentan algunas peculiaridades:

La ubicación anatomotopográfica de los ganglios linfáticos es similar a lo descrito en la literatura para otras especies, así como lo descrito por Carrasco (1968) y Gambirazio (1967) sobre la ubicación de los centros linfáticos superficiales del miembro pélvico y miembro anterior de la alpaca, respectivamente. Los ganglios mediastínicos anteriores y traqueobronquiales fueron difíciles de ubicar, mientras que los ganglios mesentéricos, constan principalmente de varias unidades pequeñas e independientes pero fueron ubicados con relativa facilidad a diferencia de lo descrito por Murray (1989) quien refiere que los ganglios linfáticos de los "lamoides" son pequeños y algo difíciles de ubicar. Los ganglios linfáticos inguinales de la alpaca macho constan de una a dos unidades independientes por lado, a diferencia de lo descrito por Carrasco (1968) el cual refiere que estos ganglios constan de varias unidades pequeñas por lado, a manera de un rosario ganglionar, coincidiendo con Murray (1989) quien refiere lo mismo para los ganglios superficiales inguinales y prefemorales superficiales, sin embargo las alpacas no cuentan con ganglios prefemorales tal como lo reporta Carrasco (1968).

Los ganglios linfáticos de las alpacas muestran seudolobulaciones superficiales, a diferencia de lo descrito en otras especies como órganos arriñonados u ovalados y con una superficie lisa y regular.

Histológicamente, los ganglios linfáticos de la alpaca no muestran una clara diferenciación entre la zona cortical y médular, tal como lo reporta Bendezú (1956), a diferencia de lo descrito por Espinoza-Chueca (1965) quien concluye que los ganglios de la alpaca presentan corteza, médula y paracorteza. Por su parte Bendezú (1956), menciona que los ganglios linfáticos de la alpaca son trilobulados, sin embargo en el presente estudio se demuestra que los ganglios son estructuras únicas.

Los senos supcapsulares, trabeculares y medulares son entidades descritas para todos los ganglios como espacios continuos interconectados (Banks, 1992; Dellman, 1982; Junqueira, 1988; Ross, 1994). En el presente estudio se observó que el seno subcapsular no es continuo sino que ocupa algunos sectores bajo la cápsula y en gran parte el parénquima está bastante adherido al estroma.

Las vénulas de endotelio alto son vasos ubicables en la paracorteza de los ganglios linfáticos y en la periferia de los folículos linfoides asociados al intestino (Girard, 1995; Kimura et al., 1996; Sainte Marie y Peng, 1996; Tizard, 1992). Tizard (1992) y Rojas (1995) mencionan que el número y longitud de estas vénulas depende de la estimulación antigénica. En los ganglios de las alpacas estas vénulas están distribuidas en todo el parénquima, principalmente en zonas asociadas a la periferia de los folículos, así como en 
el tejido linfoide asociado al intestino, lo cual sugiere que estos tejidos linfoides están sometidos a constante estimulación antigénica.

El timo de la alpaca muestra tres lobulaciones sucesivas, los lóbulos mayores divididos en sub-lóbulos medianos los cuales están conformados por los lobulillos tímicos poco observables macroscópicamente y demostrables histológicamente. La literatura solo menciona dos niveles de lobulación: los lóbulos mayores (macroscopicos) conformados por los lobulillos tímicos (histologicos) confluyentes.

De La Vega (1951) describe macroscópicamente a las placas de peyer de las alpacas como estructuras prominentes e incluso crateriformes, principalmente las placas ileocecales. En el presente estudio sólo cuatro alpacas mostraron placas de peyer visibles macroscópicamente. Histológicamente todas las alpacas mostraron nódulos linfáticos aislados y placas de peyer. Dichos resultados corroboran el hecho de que las placas de peyer son permanentes y cuyo volumen está sujeto al estímulo antigénico.

Macroscópicamente el bazo de la alpaca es similar al de los demás rumiantes. Banks (1992) y Dellman (1982) describen dos tipos básicos de bazo, el defensivo, en el cual hay poco tejido muscular pero abundante tejido linfocitario (conejos, liebres y hombre), y el de almacenamiento en el cual hay muchas trabéculas y fibras musculares lisas, es relativamente grande y posee menos pulpa blanca que el primero (caballo, perro y gato). Las formas intermedias son clásicas de los rumiantes y el cerdo, tal como se demostró en la alpaca en la cual la pulpa roja no predomina sobre la pulpa blanca.

Dichos autores refieren además, que el grosor y la composición de la cápsula esplénica varían en cada especie. Los equinos poseen la cápsula más gruesa de los animales domésticos, la capa de tejido conectivo es más gruesa que la capa muscular. En el cerdo, perro y gato la cápsula es moderadamente delgada y la capa muscular es más gruesa que la capa conjuntiva. En las alpacas la cápsula esplénica es gruesa, el estrato muscular es más grueso que el estrato conjuntivo externo, además el bazo de la alpaca presenta fibras musculares lisas aisladas en el parénquima, tal como lo reporta Dellman (1982) para el cerdo, en el cual ésta es una peculiaridad.

Estos resultados sugieren que el bazo de la alpaca cumple una función inmunológica defensiva muy importante, además de estar capacitado para almacenar gran número de eritrocitos y expelerlos eficientemente en un momento, gracias a la cantidad de fibras musculares de la cápsula y del parénquima.

\section{Literatura Citada}

1. Banks J. W. 1992. Histología Veterinaria Aplicada. P. 388-415. Manual Moderno-México.

2. Bendezú W. 1956. Aspectos histológicos de la alpaca. Organos hematopoyéticos. Tesis Bachillerato. Fac. Med. Vet. Univ. Nac. Mayor de San Marcos. Lima.

3. Carrasco A. 1968. Vasos y centros linfáticos superficiales del miembro pélvico de la alpaca. Tesis bachillerato. Fac. Med. Vet. Univ. Nac. Mayor de San Marcos. Lima.

4. Dellman H.D. y E. Brown. 1980. Histología Veterinaria. P.177-198, 262269 Ed. Acribia-España.

5. De La Vega, E. 1951. Aspectos histológicos del aparato digestivo y sistema urogenital de la alpaca. Tesis bachillerato. Fac. Med. Vet. Univ. Nac. Mayor de san Marcos. Lima.

6. Espinoza-Chueca A. 1965. Estudio Microscópico de los ganglios linfáticos de la alpaca. Tesis Médico Veterinario. Fac. Med. Vet. Univ. Nac. Mayor de San Marcos. Lima.

7. Gambirazio, C. 1967. Vasos Linfáticos superficiales y centro linfático del miembro anterior de la alpaca. Tesis Bachillerato. Fac. Med. Vet. Univ. Nac. Mayor de San Marcos. Lima. 
8. Girard J.P. y T.A. Springer. 1995. High endothelial venules (HEVs): specialized endothelium for lymphocyte migration. Immunol Today, 16: 9, 44957

9. Junqueira L.C. y J. Carneiro. 1988. Histología Básica. 3 th edición. p. 285306, 324-333. Ed. Salvat: España.

10. Kimura M., K. Tolya y K. Kuki. 1996. Laboratory suncus: a new model animal for tonsil research. Acta Otolaryngol Suppl (Stockh) Japan. 523: 20-4

11. Murray, E.F. 1989. Medicine and surgery of south american camelids. Llama, alpaca, vicuña, guanaco. 1 th edition. Iowa- USA.
12. Roitt I., J. Brostoff y D. Male. 1997. Inmunología. 4 th edición. p. 3.1-3.11 Ed.Harcout Brace: Madrid.

13. Rojas M.W. 1995. Inmunología. 10 th Ed. P. 59-75. CIB: Colombia.

14. Ross M.H., E. Reith y L. Romrell. 1994. Histología. 2 th edición p. 301334. Ed. Médica panamericana: México.

15. Sainte Marie G. y F.S. Peng. 1996. High endothelial venules of the rat lymph node. A review and question: is their activity antigen specific? Anat Rec. 245:4 593-620

16. Tizard I. 1992. Inmunología Veterinaria. 4 th edición. p. 60-79 Ed. Interamericana Mc Graw-Hill: México. 